\title{
ANÁLISIS PROXIMAL Y CUANTIFICACIÓN DE ANTOCIANINAS TOTALES EN Zea mays VARIEDAD MORADA SOMETIDO A DIFERENTES PROCESOS DE SECADO
}

\author{
Valle Campos Manuel ${ }^{\mathrm{a}}$, García Ceccarelli Jorge ${ }^{\mathrm{a}}$, Laos Anchante Doris ${ }^{\mathrm{a}}$, \\ Yarasca Carlos Eliades ${ }^{\mathrm{b}}$, Loyola Gonzales Eddie ${ }^{\mathrm{a}}$, Surco-Laos Felipe ${ }^{\mathrm{a}^{*}}$
}

\section{RESUMEN}

El Zea mays, variedad morada, es una planta oriunda de América que tiene el grano y la coronta de color morado, gracias a las antocianinas que son los pigmentos que le confieren este color. El presente trabajo tuvo como objetivo analizar la influencia de los diversos procedimientos de secado del maíz morado que ejecutan los productores del valle de Ica, sobre la composición químico proximal y el contenido de antocianinas totales. Se aplicó 5 procedimientos de secado, los que se codificaron de T1 a T5 y se analizó las corontas y los granos por separado; se observa que los diferentes tratamientos no afectan significativamente la composición químico proximal de la coronta ni del grano; sin embargo, los tratamientos 3 y 4 preservaron un mayor contenido de antocianinas en la coronta (1513 y $1516 \mathrm{mg} / 100 \mathrm{~g})$, mientras que el tratamiento 2 preservó un mayor contenido de antocianina en el grano $(907,1$ $\mathrm{mg} / 100 \mathrm{~g})$.

Palabras clave: Zea mays, secado, antocianinas, composición proximal

\section{PROXIMAL ANALYSIS AND QUANTIFICATION OF TOTAL ANTHOCYANES IN Zea mays VARIETY DWELLED SUBMITTED TO DIFFERENT DRYING PROCESSES}

\begin{abstract}
The Zea mays purple variety is a plant native to America that has purple grain and crown, thanks to the anthocyanins that are the pigments that give this color. The objective of this work was to analyze the influence of the various drying procedures of the purple maize carried out by the producers of the Ica valley, on the proximal chemical composition and the content of total anthocyanins. 5 drying procedures were applied, which were coded from T1 to T5 and the coronas and grains were analyzed separately; it is observed that the different treatments do not significantly affect the chemical composition of the crown
\end{abstract}

\footnotetext{
a Facultad de Farmacia y Bioquímica, Universidad Nacional San Luis Gonzaga de Ica

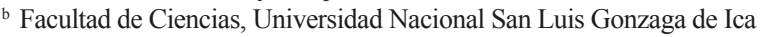

*Felipesurco@gmail.com
} 
or the grain; however, treatments 3 and 4 preserved a higher content of anthocyanins in the crown (1513 and $1516 \mathrm{mg} / 100 \mathrm{~g}$ ), while treatment 2 preserved a higher content of anthocyanin in the grain $(907,1 \mathrm{mg} / 100 \mathrm{~g})$.

Key words: Zea mays, dried, anthocyanins, proximal composition

\section{INTRODUCCIÓN}

Zea mays L. es una planta oriunda de América, se cultiva en las costas de casi todo el continente americano hasta las tierras altas de alrededor de $4000 \mathrm{msnm}$ en los Andes; se siembra en las regiones secas con una precipitación media inferior a $400 \mathrm{~mm}$ y en regiones con precipitación superior a $4000 \mathrm{~mm}$. Constituyó uno de los principales alimentos de las numerosas tribus indígenas en la época precolombina, a la variedad morada (maíz morado) se le atribuyen diversas propiedades medicinales ${ }^{1,2}$. En el Perú su consumo es popular y masivo en forma de chicha morada y mazamorra morada ${ }^{3}$.

La coloración morada que presentan las plantas, las corontas y el pericarpio de los granos, son el resultado de un complejo trabajo de muchos genes ubicados en diferente cromosomas, lo que da como resultado la formación de pigmentos antocianicos de diferentes colores ${ }^{4}$.

En los últimos años se han descrito muchas propiedades benéficas del Zea mays L, entre las que se encuentra su capacidad antihipertensiva, hipolipemiante, hipoglicemiante y antioxidante. Todas estas propiedades se atribuyen a su alto contenido de antocianinas, en especial cianidina-3-glicósido, la antocianina que el Zea mays L. variedad morado tiene en mayor cantidad y que le otorga su color característico ${ }^{5,6}$.

El distrito de Santiago es uno de los distritos de la provincia de Ica, en donde los pequeños agricultores se dedican al cultivo del maíz morado y utilizan diferentes tratamientos de secado de las mazorcas de maíz, estos tratamientos son realizados basados en conocimientos ancestrales trasmitidos de padres a hijos durante generaciones, lo que ha ocasionado que estos agricultores tengan una agronomía de subsistencia, es decir lo que cultivan solo les alcanza para solventar sus necesidades básicas. El desconocimiento de cuál de los procesos de secado es más productivo en el rendimiento de fitonutrientes hace que los productores no puedan obtener mayores beneficios económicos ${ }^{4}$.

El presente trabajo tuvo como objetivo analizar la influencia de los diversos procedimientos de secado del maíz morado que ejecutan los productores del valle de Ica, sobre la composición químico proximal y el contenido de antocianinas totales: en busca de establecer un sistema estandarizado de tratamiento de secado en base a estudios químicos, de forma tal que el agricultor con este conocimiento pueda obtener un mayor beneficio económico. 


\section{PARTE EXPERIMENTAL}

Muestras. Se tomaron cinco plantas de maíz morado para cada tratamiento, el maíz fue cultivado en el distrito de Santiago, provincia y región de Ica. Las muestras fueron unidades aleatorias de una misma parcela sometidas a los diferentes tratamientos de secado (grano y mazorca).

Preparación de la muestra. Se separa los granos de la coronta manualmente y luego se muelen, se tamizan a través de una malla $\mathrm{N}^{\circ} 20$ y se guarda en frascos oscuros tapados herméticamente hasta su análisis ${ }^{7}$.

Métodos. Para el análisis químico proximal se utilizaron los siguientes métodos oficiales: Cenizas: AOAC 923.03 Ash7, Grasa cruda o Extracto etéreo: AOAC 920.39C7 , Proteína: AOAC 070.09 Kjeldahl Method ${ }^{7}$, Carbohidratos: Por Diferencia según Collazos 1992 ${ }^{8}$, Antocianinas totales método por diferencia de $\mathrm{pH}^{9}$.

La evaluación estadística de los resultados promedios del análisis químico proximal y antocianinas encontrados en cada tratamiento de la muestra fueron sujetos a parámetros descriptivos como la media aritmética, desviación estándar, así como a un ensayo T- student, Duncan con un nivel de probabilidad del $95 \%(\alpha=0,05)^{10}$.

\section{RESULTADOS Y DISCUSIÓN}

En la tabla 1 se describe los cinco tratamientos que refieren usan los agricultores del valle de Ica para el secado de sus cosechas de maíz morado, y como se refiere anteriormente todas las muestras se obtuvieron de una misma parcela para evitar el efecto que podría atribuirse a condiciones de cultivo.

Tabla 1. Tratamientos de secados de maíz morado

\begin{tabular}{ll}
\hline $\begin{array}{l}\text { Tratamiento de } \\
\text { secado }\end{array}$ & \multicolumn{1}{c}{ Descripción } \\
\hline $\mathrm{T}_{1}$ & Secado de la mazorca en la planta al sol por cuatro meses \\
$\mathrm{T}_{2}$ & $\begin{array}{l}\text { Secado de la mazorca en la planta al sol por cuatro meses y } \\
\text { un mes libre de la planta }\end{array}$ \\
$\mathrm{T}_{3}$ & Secado de la mazorca en la planta al sol por cinco meses \\
$\mathrm{T}_{4}$ & Secado de la mazorca en la planta al sol por seis meses \\
$\mathrm{T}_{5}$ & $\begin{array}{l}\text { Secado de la mazorca en la planta al sol por seis meses y un } \\
\text { mes libre de la planta }\end{array}$ \\
\hline
\end{tabular}


En la bibliografía analizada en el presente estudio no pudimos encontrar indicación alguna sobre diferentes procesos de secado. Como se puede apreciar existen dos posibles variables que son: el tiempo de secado y la permanencia de la mazorca en la planta. Para realización de los posteriores análisis se separó el grano de la mazorca.

En la tabla 2 podemos apreciar los resultados del análisis químico proximal de las corontas (chuzas) de los cinco tratamientos de secados empleados, se pude observar que, el de menor tiempo de secado (4 meses) es el que contiene un mayor porcentaje de humedad, lo que justificaría el menor contenido de proteína y carbohidratos, guardando correlación con el proceso mismo, mientras que en los otros tratamientos las diferencias no son significativas.

Tabla 2. Análisis químico proximal de corontas según tratamientos

\begin{tabular}{cccccc}
\hline \multirow{2}{*}{ Tratamientos } & \multicolumn{5}{c}{ Análisis Proximal (g/100g) } \\
& Humedad & Ceniza & Proteína & Grasa & C.H. \\
\hline T1 & $9,30 \pm 0,23$ & $3,37 \pm 0,09$ & $6,02 \pm 0,18$ & $0,045 \pm 0,011$ & 81,27 \\
T2 & $4,80 \pm 0,37$ & $2,05 \pm 0,11$ & $7,56 \pm 0,22$ & $0,050 \pm 0,013$ & 85,54 \\
T3 & $5,40 \pm 0.42$ & $2,82 \pm 0,21$ & $7,15 \pm 0,27$ & $0,028 \pm 0,011$ & 84,60 \\
T4 & $5,33 \pm 0,34$ & $3,42 \pm 0,05$ & $7,19 \pm 0,23$ & $0,050 \pm 0,008$ & 84,01 \\
T5 & $4,78 \pm 0.45$ & $3,07 \pm 0,16$ & $7,81 \pm 0,08$ & $0,046 \pm 0,011$ & 84,29 \\
\hline Los resultados son el promedio de tres determinaciones y su desviación estándar & \\
C.H. carbohidratos: los valores son la diferencia de los promedios según Collazos & \\
\end{tabular}

En cuanto al análisis químico proximal de las corontas, lo que queda claro es que cuando se separa la mazorca de la planta se logra un mayor secado $(\mathrm{T} 1 \rightarrow \mathrm{T} 2$ y $\mathrm{T} 4 \rightarrow \mathrm{T} 5)$. El contenido relativo de proteína lo hace un ingrediente adecuado en la preparación de alimentos balanceados para animales de inverna.

El análisis estadístico solo presentó diferencia significativa en humedad T1, en cenizas T2, en proteína $\mathrm{T} 1 \mathrm{y}$ en grasa $\mathrm{T} 3$.

En la tabla 3, de contenido de antocianinas, podemos observar dos aspectos relevantes:

- Primero: que los tratamientos T3 y T4 son los de mayor contenido de antocianinas, lo que podría de manifiesto que los procesos fisiológicos en la planta continúan y estos principios activos se siguen transfiriendo de tallos y las brácteas hacia la mazorca4, siendo el tiempo máximo de cinco meses, puesto que ambos tratamientos tienen el mismo contenido, aunque en diferentes condiciones.

- Segundo: luego de separar las mazorcas del tallo y proseguir el proceso de secado se pierde las antocianinas (T1 a T2 y T4 a T5). 
Tabla 3. Contenido de antocianinas totales en corontas según tratamiento

\begin{tabular}{ccc}
\hline \multirow{2}{*}{ Tratamientos } & \multicolumn{2}{c}{ Contenido de antocianinas totales $(\mathrm{mg} / 100 \mathrm{~g})$} \\
\cline { 2 - 3 } T1 & Base Normal & Base seca \\
T2 & $1345 \pm 84$ & $1430 \pm 92^{\mathrm{c}}$ \\
T3 & $885 \pm 76$ & $930 \pm 90^{\mathrm{a}}$ \\
T4 & $1432 \pm 139$ & $1513 \pm 147^{\mathrm{d}}$ \\
T5 & $1436 \pm 143$ & $1516 \pm 151^{\mathrm{d}}$ \\
\end{tabular}

Letras iguales no hay diferencia estadística significativa

Por lo tanto, si es de aprovechar el contenido de antocianinas en la mazorca, el mejor tratamiento estaría representado por el T3 (5 meses); sin embargo, el tratamiento T2 presenta contenido ligeramente menor de antocianinas pero es un tiempo de secado menor (4 meses).

En tabla 4, en cuanto al contenido del análisis químico proximal del grano, como se observa de una manera general, podemos indicar que no hay diferencia estadística significativa entre los diferentes tratamientos, salvo en el caso de la determinación de grasa para el tratamiento $\mathrm{T} 3$ y en proteína para los tratamientos T4 y T5 cuyos valores son ligeramente superiores a los otros.

Tabla 4. Análisis químico proximal de granos según tratamientos

Análisis Proximal (g/100g)

\begin{tabular}{cccccc}
\hline Tratamientos & Humedad & Ceniza & Proteína & Grasa & C.H. \\
T1 & $10,13 \pm 0.27$ & $2,80 \pm 0,13$ & $9,47 \pm 0,17$ & $4,16 \pm 0,22$ & 73,44 \\
T2 & $10,36 \pm 0.11$ & $2,71 \pm 0,17$ & $9,0 \pm 0,34$ & $4,48 \pm 0.17$ & 73,45 \\
T3 & $10,22 \pm 0,23$ & $2,25 \pm 0,13$ & $9,86 \pm 0,13$ & $5,17 \pm 0,26$ & 72,50 \\
T4 & $10,5 \pm 0,09$ & $2,29 \pm 0,13$ & $10,32 \pm 0,09$ & $4,16 \pm 0,29$ & 72,73 \\
T5 & $10,13 \pm 0.15$ & $2,09 \pm 0,29$ & $10,46 \pm 0.010$ & $4,33 \pm 0,18$ & 72,99 \\
\hline
\end{tabular}

C.H. Los valores son la diferencia de los promedios según Collazos ${ }^{8}$.

En cuanto al contenido del análisis químico proximal del grano, como se observa en la tabla 4, de una manera general, podemos indicar que no hay diferencia estadística significativa entre los diferentes tratamientos, salvo en el caso de la determinación de grasa para el tratamiento T3 y en proteína para los tratamientos T4 y T5 cuyos valores son ligeramente superiores a los otros. 
Por lo tanto, podemos afirmar, de manera general, que los distintos procesos de secado no afectan la calidad nutricional del grano del maíz morado, si bien los valores de proteína son mayores que los reportados en otras variedades de maíz ${ }^{11,12}$, son coincidentes con reportes de Cabieses $^{3}$.

En la tabla 5, respecto al contenido de antocianinas totales en grano de los diferentes tratamientos, podemos observar que expresados en base seca, el tratamiento que presenta un mayor contenido de antocianinas totales es T2, en el cual la muestra fue secada en la planta durante cuatro meses. A mayores meses la mazorca en la planta no contribuye a un mayor proceso de transferencia de antocianinas desde la planta al grano; muy por el contrario disminuye T3 y T4, lo que indicaría que el proceso fisiológico acabaría (muerte celular).

Sin embargo, al separar la mazorca de la planta, en una primera etapa pareciera continuar la transferencia de antocianinas desde las brácteas (panca) al grano, que luego a mayores meses disminuye.

Tabla 5. Contenido de antocianinas totales en granos según tratamiento

\begin{tabular}{ccc}
\hline & \multicolumn{2}{c}{ Contenido de antocianinas totales $(\mathrm{mg} / 100 \mathrm{~g})$} \\
\hline Tratamientos & Base Normal & Base seca \\
T1 & $682,1 \pm 69$ & $758,9 \pm 76^{\mathrm{b}}$ \\
T2 & $813,1 \pm 83$ & $907,1 \pm 89^{\mathrm{c}}$ \\
T3 & $622,8 \pm 66$ & $740,6 \pm 70^{\mathrm{b}}$ \\
T4 & $459,5 \pm 54$ & $694,7 \pm 57^{\mathrm{a}}$ \\
T5 & $553,6 \pm 61$ & $616,0 \pm 64^{\mathrm{a}}$ \\
\hline
\end{tabular}

Letras iguales no hay diferencias estadísticas significativas

Por lo tanto, podemos afirmar que los diferentes procesos de secado sí afecta considerablemente el contenido de antocianinas en el grano del maíz, otros estudios han reportado diferentes concentraciones de antocianinas y que varían con la variedad ${ }^{13,14}$.

\section{CONCLUSIONES}

Los agricultores del valle de Ica aplican cinco diferentes tratamientos para el secado del maíz morado. La composición químico proximal no se ve afectada por los distintos tratamientos de secados. Los tratamientos 3 y 4 preservan un mayor contenido de antocianinas en la coronta. El tratamiento 2 preserva mayor contenido de antocianina en el grano. 


\section{REFERENCIAS BIBLIOGRÁFICAS}

1. Palacios V. Plantas medicinales nativas del Perú. Lima: Consejo Nacional de Ciencia y Tecnología; 1997.

2. Fernández Pola J. Recetario de plantas medicinales. Barcelona: Ediciones Omega SA; 1992.

3. Cabieses F. Cien siglos de pan: 10,000 años de alimentación en el Perú. Lima: Universidad de San Martín de Porres; 2004.

4. Salgado J, Menguan E. Determinación de compuestos fenólicos en estilos, estigmas, estambres y brácteas en la especie vegetal Zea mays L. variedad morada. [Tesis de Bachiller]. Lima: Facultad de Farmacia y Bioquímica, Universidad Nacional Mayor de San Marcos; 2000.

5. Nakatani N, Fukuda H, Fuwa H. Major anthocyanin of Bolivian purple corn (Zea mayz L.). Agric Biol Chem Tokyo. 1979; 43: 389-91.

6. Pedreschi R, Cisneros-Zevallos L. Phenolic profiles of Andean purple corn (Zea mays L.). Food Chem. 2007; 100(3): 956-63.

7. AOAC. Official Methods of Analysis 19th Edition. Rockville, Maryland, USA: AOAC International; 2012.

8. CENAN. Tabla Peruana de Composición de los Alimentos. Lima: MINSA; 2009

9. Fossen T, Slimestad R, Andersen $\varnothing$. Anthocyanins from maize (Zea mays) and reed canarygrass (Phalaris arundinacea). J Agric Food Chem. 2001; 49: 2318-2321.

10. Glantz S. Bioestadística. $6^{\circ}$ Ed. México: Ed. Mc. Graw-Hill/Interamericana Editores, S.A de C.V.; 2005. 73-81.

11. Mendoza MG. Las antocianinas del maíz: su distribución en la planta y producción. [Internet]. 2008 [citado 12 abril 2017]. Disponible en: http://www.lareferencia.info/ vufind/Record/MX_8e3387f92494a9ddc7b2057dd5e5

12. Kirk R, Sawyer R. Composición y Análisis de los Alimentos de Pearson. 2da edición. México D.F.: Editorial Patria; 2011.

13. Montilla C, Hillebrand S, Antezana A, Winterhalter P. Soluble and bound phenolic compounds in different Bolivian purple corn (Zea mays L.) cultivars. J Agric Food Chem. 2011; 59: 7068-7074.

14. Salinas M, Soto M, Martínez F, González V, Ortega R. Análisis de antocianinas en maíces de grano azul y rojo provenientes de cuatro razas. Rev Fitotec Mex. 1999; 22: $161-174$. 